\title{
(Dé)voy(ag)er : le rire de l'écrivain romantique en voyage, ou le genre subverti
}

\section{Alain Guyot}

\section{Q OpenEdition}

\section{Journals}

Édition électronique

URL : http://journals.openedition.org/recherchestravaux/271

DOI : 10.4000/recherchestravaux.271

ISSN : 1969-6434

Éditeur

UGA Éditions/Université Grenoble Alpes

\section{Édition imprimée}

Date de publication : 30 mai 2005

Pagination : 127-137

ISBN : 0151-1874

ISSN : 0151-1874

\section{Référence électronique}

Alain Guyot, «(Dé)voy(ag)er : le rire de l'écrivain romantique en voyage, ou le genre subverti », Recherches \& Travaux [En ligne], 67 | 2005, mis en ligne le 30 septembre 2008, consulté le 08 septembre 2020. URL : http://journals.openedition.org/recherchestravaux/271 ; DOI : https://doi.org/ 10.4000/recherchestravaux.271 


\section{(Dé) voy(ag)er : le rire de l'écrivain romantique en voyage, ou le genre subverti}

Alors qu'il est en train de faire le récit de sa traversée de la Morée, et plus précisément d'une nuit passée au village de Lerne, couché «sur un fumier de brebis, lieu le moins sale et le moins humide que nous pûmes trouver", Chateaubriand ajoute cette notation:

Je serais en droit de faire une querelle à Hercule, qui n'a pas bien tué l'hydre de Lerne: car je gagnai dans ce lieu malsain une fièvre qui ne me quitta qu'en Égypte. (Itinéraire, p. 834)

Guère mieux loti en Espagne, son fils spirituel Custine se plaint d'une auberge à San Lucar "où l'on n'a rien à manger tandis qu'on y est mangé soimême par une armée d'ennemis dégoûtants" (L'Espagne sous Ferdinand VII, p. 369), tandis que Dumas déplore la piètre qualité des lits suisses, sur lesquels

[...] on étend, en le décorant du titre de drap, une espèce de nappe si courte, qu'elle ne peut ni se replier à l'extrémité inférieure, sous le matelas, ni se rouler à l'extrémité supérieure, autour du traversin, de sorte que les pieds et la tête en peuvent jouir alternativement, il est vrai, mais jamais les deux à la fois. (Impressions de voyage en Suisse, t. I, p. 9I)

Hugo compare de son côté les assiettes qu'il trouve en I836 dans les auberges bretonnes à des "formations" géologiques:

Il faudrait pénétrer plusieurs couches de je ne sais quoi pour arriver à la faïence. $\mathrm{Si}$ les puces marchaient, elles y laisseraient très certainement l'empreinte de leurs petits pieds. (Voyages, p. 573)

Gautier, pour sa part, se gausse d'un Wallon monté dans la diligence qui le conduit à Bruxelles et muni d'une immense tabatière, pareille à

I. Pour les références abrégées, voir la bibliographie en fin d'article. 
une table à douze couverts [...] dont les deux charnières poussaient, en tournant sur elles-mêmes, des miaulements plus affreux que ceux de vingt chats écorchés vifs (Un tour en Belgique, p. Iо)

Le marchand de fer voyageur de Stendhal s'amuse à raconter l'histoire d'un jeune et beau Grenoblois "fort instruit et parfaitement aimable»:

Il écrivait ses confessions, et avec tant de grâce que son confesseur le lui a défendu.

Vous jouissez une seconde fois de vos péchés en les écrivant ainsi, dites-les-moi de vive voix. (Mémoires d'un touriste, p. 388)

George Sand et Nerval se plaisent à raconter leurs mésaventures: l'une, après avoir contemplé, à la nuit tombée, le massif du Mont-Blanc par la fenêtre de sa chambre, occasion d'une description fort poétique, se fait dans le noir "une bosse à la tête contre l'angle du mur»:

C'est ce qui me dégoûta de faire des métaphores tous les jours subséquents. Mes amis eurent l'obligeance de s'en déclarer singulièrement privés. (Lettres d'un voyageur, p. 902)

L'autre, victime d'une insuffisante maitrise de la langue arabe, s'aperçoit qu'au lieu de réprimander le mousse du bateau qui le mène au Liban, il lui a fait des avances à son insu, se faisant ainsi passer aux yeux du capitaine pour ce qu'il ne veut point être (Voyage en Orient, p. 296-297). Il n'est pas jusqu’à Mérimée, pourtant corseté par son austère fonction d'inspecteur des monuments historiques, qui ne s'autorise, dans les rapports qu'il adresse à son ministre de tutelle, à railler ici «l'obscénité de quelques figures» ornant les chapiteaux d'une église ou à raconter là l'histoire de ce berger corse qui

[...] trouva un jour une [...] statue avec cette inscription: Girami, e vedrai... [...] à grand-peine on l'avait retournée, et trouvé la fin de l'inscription: il rovescio. C'est la contrepartie de l'histoire du licencié Gil Perez. (Notes d'un voyage en Corse, p. 53 et $3 \mathrm{I}^{2}$

On pourrait multiplier les exemples. Qu'ils se moquent d'eux-mêmes ou des autres, qu'ils s'émerveillent ou qu'ils s'indignent, qu'ils se réjouissent ou se lamentent, qu'ils satirisent, ironisent ou simplement «humorisent», esprit léger ou comique "hénaurme», les écrivains en voyage au temps du Romantisme passent beaucoup de temps à rire, et surtout à faire rire les lecteurs de leurs récits. Ils ne sont certes pas les premiers: déjà, en son temps, Jean de Léry, explorant le Brésil, s'amusait de voir que son nom signifiait "grosse huître" dans la langue des sauvages "Toüoupinambaoults» (Histoire d'un voyage, p. 174). Bougainville n'hésita pas de son côté à dauber les jésuites du

2. «Retourne-moi et tu verras l'arrière.» 
Paraguay, en quelques pages cinglantes qui constituent un modèle du genre ironique. On avait même vu fleurir au XVII ${ }^{e}$ siècle le genre du «voyage amusant", inventé par Chapelle et Bachaumont, et dont le succès ne se démentira pas durant plus de deux cents ans ${ }^{3}$. Balançant depuis toujours entre science et littérature, entre la nécessité d'informer et le désir de plaire4, le récit de voyage, quelle que soit la forme qu'il choisisse, a toujours été attiré par la dimension comique, qu'elle lui offre l'occasion d'atténuer le choc suscité par la rencontre avec la nouveauté et l'altérités ou qu'elle lui permette, au même titre que les récits d'aventure, de raviver l'intérêt d'un lecteur vite lassé par des chorographies fastidieuses, déjà tournées en dérision par Sterne dans son Voyage sentimental (1768). Mais jamais on n'avait vu encore le récit de voyage à tel point envahi, en quantité comme en qualité, par le rire, qui ne se cantonne plus désormais aux seuls voyages «amusants» ou à leur filiation «humoristique $^{6} »$. Il y a loin en effet de la badinerie de bon goût et des fantaisies burlesques, prônées par ces derniers, à cette charge du jeune Flaubert voyageant en Bretagne en I847, en compagnie de son ami Du Camp:

Je donnerais bien le Villemain complet que j’ai acheté dans mon enfance (action insensée qui ne m’a pas fait interdire, ce qui prouve la débonnaireté de ma famille); je donnerais aussi le cours de M. St. Marc-Girardin que je conserve comme dit René "pour m'ôter à l'avenir tout mouvement de joie»; j'y ajouterais même une vieille paire de babouches marocaines qui, l'été, m'est très commode, et de plus mes droits de citoyen, l'estime de mes compatriotes, et le reste d'une bouteille de beau vernis qui commence à s'épaissir, oui, je donnerais tout cela de bon cœur - et sur l'heure - pour savoir le nom, l'âge, la demeure, la profession et la figure du Monsieur qui a inventé pour les statues du musée de Nantes, des feuilles de vignes en fer-blanc, qui ont l'air d'appareils contre l'onanisme. L'Apollon du Belvédère, le Discobole et un joueur de flûte sont enharnachés de ces honteux caleçons métalliques qui reluisent comme des casseroles. On voit d'ailleurs que c'est un ouvrage médité de longtemps et exécuté avec amour. C'est escalopé sur les bords et enfoncé avec des vis dans les membres des pauvres plâtres, qui s'en vont écaillés de douleur. Par ce temps de bêtises plates qui court, au milieu des stupidités normales qui nous encombrent, il est réjouissant, ne fût-ce que par diversion, de rencontrer au moins une bêtise échevelée, une stupidité gigantesque! Malgré tous mes efforts, je ne suis parvenu à me rien figurer sur le créateur de cette pudique immondicité. J'aime à croire que le conseil municipal en

3. La Bibliothèque nationale réédite encore sous ce titre, en 1906, le fameux Voyage d'Encausse (I660), le Voyage en Languedoc de Lefranc de Pompignan (I745) et le Voyage de Paris à Saint-Cloud par mer de Néel (1748). Sur ce sujet, voir Bideaux et Racault.

4. Voir Chupeau, p. 54I, 544 sq., et Le Huenen, p. 47-48.

5. Voir Guentner, p. 304.

6. Pour une enquête sur les prolongements du genre du "voyage amusant» aux XVIII e et XIX $^{e}$ siècles, en particulier sur le renouveau qu'il connaît sous l'influence de Sterne ou de Maistre, voir Sangsue. 
entier y a pris part, que MM. les ecclésiastiques l'avaient sollicitée, et que les dames l'ont trouvée convenable. (Par les champs, p. 177-I79)

On a clairement affaire ici à une satire de la pruderie, provinciale et louisphilipparde, qui fera les beaux jours du roman flaubertien. L'écrivain en herbe fait flèche de tout bois rhétorique pour parvenir à ses fins, de la période initiale, qui crée une attente sans cesse prolongée par de nouvelles parenthèses suscitant un effet comique au second degré, aux rapprochements incongrus, parallélismes syntaxiques, alliances de termes, oxymores, analogies tirant toujours le comparé vers le grotesque hyperbolique ou le monstrueux, sans oublier le décalage ironique entre le sujet attendu (une description d'œuvres d'art) et celui qui est réellement traité, les feuilles de vigne affublant les pauvres statues, désormais réduites, par le jeu de la synecdoque, à de simples "plâtres»... Si la charge en question n'a pas la pureté de celle d'un Juvénal, l'intention satirique reste indéniable. Elle préside même à l'ensemble de cet ouvrage de jeunesse que Flaubert écrivit en collaboration avec son ami Du Camp, mais ne voulut jamais publier. Alors que ce dernier privilégie les bons mots, aphorismes ou autres «axiomes» dont la vulgarité confine parfois au cliché7, Flaubert paraît trouver dans la satire le moyen de "traquer le dérisoire» et de manifester sa réprobation à l'égard de spectacles répulsifs à ses yeux. Raymonde Debray-Genette a bien montré comment, indigné qu'il est par la pompe ostentatoire d'une procession à Quimper ${ }^{8}$, il a recours à une multitude de procédés comiques pour tourner en dérision l'absence de spiritualité qui marque la manifestation'. Ailleurs, il s'en prend à ces lieux à la réputation surfaite, véritables clichés de la sensiblerie du temps, que les guides de voyage l'amènent à visiter - bien inutilement à son goût -, comme la grotte d'Héloïse, près de Clisson, célébrée avec un romantisme béat par «Monsieur Richer, auteur d'un voyage dans la Loire-inférieure»:

Eh bien! je l'avoue je ne suis pas comme Monsieur Richer ni comme Héloïse ; j'ai senti peu de chose, je n'ai admiré que les arbres, trouvant que la grotte qu'ils ombragent serait très congruente pour y déjeuner, l'été, en compagnie de quelques amis et d'Héloïses quelconques, d'autant que la proximité de l'eau permettrait d'y mettre rafraîchir les bouteilles, et je n'ai rien rêvé du tout [...]. (Par les champs, p. 195) ${ }^{10}$

7. «[L]e veau est au filet de bœuf, ce que Sainte-Beuve est à Victor Hugo» (p. 226), «Les routes sont comme les femmes, il faut beaucoup d'argent pour les entretenir.» (p. 635)

8. «[C]a fait une impression de prostitution [...] on est révolté, q[uoi]qu’on ne veuille pas l'etre [sic] (carnets du voyage de Flaubert en Bretagne dans Par les champs, p. 699).

9. Debray-Genette, p. 248-249. Voir aussi Guentner, p. 290 sq.

ı. Voir aussi la désinvolture du "touriste» de Stendhal à l'égard de la fontaine de Vaucluse (Mémoires d'un touriste, p. 158). 
De l'art de transformer une pastorale en Déjeuner sur l'herbe avant l'heure... Plutôt que la polémique ou l'invective, auxquelles Chateaubriand ou Lamartine ont si volontiers recours pour sensibiliser leur public à des causes entendues (le joug ottoman sur l'Orient, par exemple), Flaubert adopte, comme Gautier et tant d'autres, un ton satirique pour marquer sa réaction de sujet écrivant à l'égard de la bêtise ou de la médiocrité - quitte à «choquer le bourgeois» en préférant aux descriptions des monuments de Brest celles du bagne, d'un cirque et du quartier "chaud ${ }^{\mathrm{II}}$ ». En fonction du système de valeurs qui lui est propre, le voyageur cherche, tel Molière, à «mettre les rieurs de son côté " pour stigmatiser un comportement ou simplement épingler un personnage, si haut placé soit-il, à l'instar de Hugo dressant le portrait d'un conducteur de diligence au cours de son voyage dans les Pyrénées:

Ce brave homme ressemblait parfaitement à M. de Rambuteau. En le contemplant, je me disais qu'il suffirait peut-être de le raser pour en faire un préfet de la Seine, et qu'il suffirait aussi que M. de Rambuteau ne se rasât plus pour faire un excellent directeur de diligence. (Voyages, p. 758)

Le comique des récits de voyage romantiques n'est pourtant pas réductible à la seule stratégie de la satire. Elle est pratiquement absente chez le Chateaubriand de l'Itinéraire: ses sarcasmes face aux tombeaux des rois d'Espagne à l'Escurial ${ }^{12}$ ne peuvent se justifier que par l'espèce de lassitude que rencontre l'écrivain à l'extrême fin de son voyage - et surtout de son récit ${ }^{13}$. Quand par ailleurs il se moque, ce n'est guère que de lui-même ou de l'un de ses proches. Il raille ainsi sa propre tendance aux rêves de gloire au moment où il quitte Athènes: s'imaginant avoir reçu l' "Attique en souveraineté", il se rêve en bâtisseur de routes et de ports, bienfaiteur de l'humanité, protecteur des arts et des lettres, en un mot restaurateur de la grandeur d'Athènes, avant de sortir du songe pour "[s]e retrouv[er] Gros-Jean comme devant» (Itinéraire, p. 894). Il dresse en outre un étrange portrait du guide qui l'a accompagné au cours de sa traversée de la Grèce, au moment où il va le quitter:

Ma dernière visite à Smyrne fut pour Joseph: Quantum mutatus ab illo! Était-ce bien là mon illustre drogman? Je le trouvai dans une chétive boutique, planant et battant de la vaisselle d'étain. Il avait cette même veste de velours bleu qu'il

II. Voir p. 498 sq. Faut-il y voir l'influence de Gautier, qui, pour se moquer de la mode des excursions romantiques, s'amusait à représenter, dans son Voyage hors barrières, un abattoir ou des combats d'animaux aux lisières de Paris?

I2. «[...] toute cette poussière est étiquetée et rangée en ordre, comme les curiosités d'un muséum» (p. I213).

I3. "Je n'ai plus rien à dire aux lecteurs; il est temps qu'ils rentrent avec moi dans notre commune patrie.» (Itinéraire, p. I2I2) Peut-être faut-il également interpréter ainsi les amères remarques de Flaubert sur Rennes, dernière étape de son voyage en Bretagne et ultime page par lui rédigée dans le récit (Par les champs, p. 63I) : voir Debray-Genette, p. 247. 
portait sur les ruines de Sparte et d'Athènes. Mais que lui servaient ces marques de sa gloire? Que lui servait d'avoir vu les villes et les hommes, mores hominum et urbes. Il n'était pas même propriétaire de son échoppe! (p. 924)

Le plus intéressant - et le plus drôle - est peut-être moins ici le décalage entre l'apparence et la réalité du drogman que celui qui s'établit entre ce personnage et la manière dont il est représenté, puisque Chateaubriand se sert alors de l'appareil discursif et rhétorique qu'il a coutume d'utiliser lorsqu'il évoque les monuments et les ruines: citations (Virgile et Horace), questions rhétoriques, thématique du même et de l'autre, de la déploration et de la gloire. La parodie peut certes se révéler un instrument particulièrement redoutable sous la plume de l'écrivain voyageur satiriste ${ }^{\mathrm{I4}}$, mais est-ce bien le but visé dans ce portrait héroï-comique? Ne s'agirait-il pas plutôt d'un souvenir du "voyage amusant", voire d'une manifestation discrète d'autodérision, que ne dédaigne pas le futur auteur des Mémoires d'outre-tombe?

Il n'est pas sûr non plus que le goût du monstrueux ait toujours partie liée avec la raillerie morale, comme lorsque Gautier fait le portrait d'

[...] un grand vieillard, maigre comme un lézard qui a jeûné six mois, et pour ainsi dire momifié, si sec, que s'il eût mouché la chandelle avec ses doigts, il se serait infailliblement allumé. Son front peaussu avait plus de fossés et de contrescarpes qu' une ville fortifiée à la Vauban. (Un tour en Belgique, p. 8)

De même, lorsque Flaubert s'amuse à décrire, avec force exclamations, un chapeau gigantesque aperçu à Plouharnel, pareil à «un couvercle de chaudière à vapeur surmonté d'une colonne» (Par les champs, p. 3II), on sent clairement que le plaisir de l'écriture a pris le pas sur l'intention moqueuse.

C'est donc ailleurs qu'il faut chercher les raisons de cette prolifération du comique dans le récit de voyage romantique. Est-ce un hasard si à ce moment correspond précisément son "entrée en littérature», pour reprendre les mots de Roland Le Huenen? De fait, ce dernier a bien montré comment « [c]'est la littérature dès lors qui fixera au voyage son objet et sa finalité, en même temps que la figure du voyageur se confondra de plus en plus avec celle de l'écrivain " (Le Huenen, p. 5I). Alors qu'il n'avait abordé jusque-là le genre qu’occasionnellement ou dans un cadre "amusant», tels Racine ou La Fontaine, l'écrivain se retrouve à

[...] occuper, et cela de manière quasi exclusive, le poste de producteur que se partageaient jusqu'alors navigateurs, géographes, missionnaires, marchands, ambassadeurs, militaires et commis de l'État, qui avaient tous en commun d'appartenir à des champs d'activités autres que celui de la littérature. (Ibid.)

I4. Voir par exemple la manière dont Flaubert parodie le genre de la description de tableau pour critiquer une série de gravures bien-pensantes exposées dans une auberge de Cancale (Par les champs, p. 596-599). 
La mission dont il se charge semble d'autant plus redoutable que les progrès des Lumières avaient conduit à une réhabilitation d'un récit de voyage "sérieux", à caractère scientifique, voire encyclopédique, à l'opposé de l'écriture littéraire ${ }^{\mathrm{I}}$. Dans la préface de l'Itinéraire, Chateaubriand est l'un des tout premiers à mettre en évidence la différence radicale entre ses projets en la matière et ceux de ses prédécesseurs:

Je prie donc le lecteur de regarder cet Itinéraire, moins comme un Voyage que comme des Mémoires d'une année de ma vie. Je ne marche point sur les traces des Chardin, des Tavernier, des Chandler, des Mungo Parck, des Humboldt: je n'ai point la prétention d'avoir connu des peuples chez lesquels je n'ai fait que passer. Un moment suffit au peintre de paysage pour crayonner un arbre, prendre une vue, dessiner une ruine; mais les années entières sont trop courtes pour étudier les mœurs des hommes, et pour approfondir les sciences et les arts. (p. 70I)

Il n'en reste pas moins soucieux de ses « devoirs d'écrivain » envers un genre bien établi :

Toutefois je sais respecter le public, et l'on aurait tort de penser que je livre au jour un ouvrage qui ne m’a coûté ni soins, ni recherches, ni travail [...]. (Ibid.)

L'écrivain romantique en voyage se retrouve donc aux prises avec un genre contraignant, fort anciennement établi, mais qu'il va progressivement faire évoluer de l'intérieur, pour opérer un véritable "détournement générique». Dans le prolongement du «style enjoué» propre au "voyage amusant ${ }^{\mathrm{I} 6}$ ", il va ainsi diversifier les tons et les discours afin de soutenir l'intérêt de la lecture, et le comique ou la satire pourront contribuer à l'occasion à balancer un passage trop austère, trop dramatique ou trop sentimental ${ }^{17}$. Comment s'étonner alors de voir Chateaubriand dénouer la tension suscitée par le récit d'une traversée particulièrement périlleuse de l'Eubée en mettant précisément en scène le personnage d'écrivain sérieux qu'il entend jouer depuis sa préface?

Je ne prétends point faire valoir mes travaux, qui sont très peu de chose; mais j'espère cependant que quand on me verra m'arracher à mon pays et à mes amis, supporter la fièvre et les fatigues, traverser les mers de la Grèce dans de petits bateaux, recevoir les coups de fusil des Bédouins, et tout cela par respect pour le

I5. "[...] le genre des voyages appart[ien]t à l'histoire et non au roman.» (Volney, Voyage en Syrie, p. 23). Voir à ce sujet Duchet I971, p. 98 sq.; Le Huenen, p. 48; Berchet, p. 5.

I6. «[...] de même qu'un voyageur qui marcherait longtemps dans un pays plat qui ne lui fournirait toujours qu'une même vue, ne manquerait pas à la fin de s'en lasser; ainsi il ne faut point douter qu'on ne s'ennuyât de lire un Voyage dont le discours n'aurait partout qu'un même style» ("Au lecteur», Nouveau Recueil de poésies françaises, Paris, 1656).

17. «Dans un ouvrage du genre de cet Itinéraire, j’ai dû souvent passer des réflexions les plus graves aux récits les plus familiers: tantôt m'abandonnant à mes rêveries sur les ruines de la Grèce, tantôt revenant aux soins du voyageur, mon style a suivi nécessairement les mouvements de ma pensée et de ma fortune." (Itinéraire, p. 702) Voir Guentner, p. 290. 
public, et pour donner à ce public un ouvrage moins imparfait que le Génie $d u$ christianisme; j'espère, dis-je, qu'on me saura quelque gré de mes efforts. (p. 9159I6)

Dans la même optique, il s'agit d'aménager le caractère didactique, souvent rébarbatif, du récit de voyage traditionnel, et instruire le lecteur en l'amusant n'est pas le moindre des mots d'ordre chez Hugo en excursion dans les Pyrénées, entre autres:

Comme nous sortions de Tartas, un lièvre énorme sortit d'un taillis voisin et traversa la chaussée, puis s'arrêta à une portée de pistolet dans une prairie et regarda la diligence. Cette bravoure des lièvres dans ce pays tient sans doute à ce qu'ils savent que ce sont eux qui ont donné leur nom à la maison d'Albret. La fierté les a pris, et ils se comportent, le cas échéant, en lièvres gentilshommes. (Voyages, p. 761-762)

Le savoir érudit sur les origines de la maison d'Albret est transformé, par le biais d'une intégration narrative, en une fable en raccourci, qui parodie aimablement La Fontaine. Mais il s'en faut que tous les écrivains voyageurs du temps aient la même attitude à l'endroit de la nécessaire dimension cognitive propre au genre viatique. Si, dans un passage bien connu de Par les champs et par les grèves, Flaubert trouve le moyen de présenter un exposé d'archéologie celtique consacré aux menhirs de Carnac en faisant défiler les interprétations émises à leur sujet pour mieux les tourner en dérision ${ }^{18}$, il manifeste beaucoup plus de réticence dans les lignes qu'il consacre à l'histoire de Quimper (p. 384-386). Sans doute cherche-t-il déjà, bien avant Bouvard et Pécuchet, à dénoncer

[...] à la fois la prétention à l'exhaustivité que pourrait fournir la science documentaire et l'immense écart, où s'engouffre une hypothétique exactitude, entre un abord sapientiel et un abord existentiel de tout objet descriptif. (DebrayGenette, p. 239)

Ces clins d'œil pleins d'ironie qui ponctuent tant de récits de voyage lorsqu'il s'agit d'insérer un morceau didactique ${ }^{19}$ ne seraient-ils pas en réalité le moyen pour l'écrivain de dénoncer une fiction: celle de l'adéquation du monde et de la parole? Le voyageur romantique a en effet perdu la naïveté du rapport au monde qu'avaient pu connaître ses lointains prédécesseurs explorant

I8. P. 256 sq. Raymonde Debray-Genette se demande s'il faut prendre au sérieux cette "longue prétérition [...] "hénaurme" et bravache» (p. 239), mais on se souviendra qu'il s'agit tout de même du seul morceau publié du vivant de l'auteur (dans L'Artiste du i8 avril I858).

19. Voir, entre autres, George Sand se proposant de donner, dans son Hiver à Majorque, « une description très succincte de la grande Baléare, dans la forme vulgaire d'un article de dictionnaire géographique [...] et, pour ne pas me départir de mon rôle de voyageur, je commence par déclarer qu'il est incontestablement supérieur à tous ceux qui le précèdent." (p. IO4OI04I) Sur le refus du savoir dans le voyage «humoristique», voir Sangsue, p. II4I. 
des terres nouvelles: celles qu'il visite sont défrichées depuis longtemps, et les textes ou les images lui ont déjà fourni matière à savoir - ou à rêverie - à leur sujet. La seule chose que lui autorise son voyage ne peut donc être au mieux qu'une reconnaissance, accompagnée, au pire, d'une désillusion, lorsque ses souvenirs de lecture sont déçus ou qu'en lui se fait jour le sentiment d'une incapacité à décrire le réel ${ }^{20}$. Le recours au comique peut alors se révéler un moyen de signaler l'impossibilité d'un rapport direct au monde, tout en atténuant peut-être la souffrance entraînée par cette prise de conscience:

[...] je ne conçois pas comment la nature pouvait avoir l'air aussi peu naturelle et ressembler autant à une mauvaise tenture de salle à manger. Je ne sais si l'habitude de voir des tableaux m’a faussé les yeux et le jugement, mais j'ai éprouvé assez souvent une sensation singulière en face de la réalité; le paysage véritable m’a paru peint et n'être, après tout, qu'une imitation maladroite [...]. (Gautier, Un tour en Belgique, p. 6)

Autant de raisons pour que le genre du récit de voyage d'écrivain, dans les années i830, n'ait plus honte de l'autonomie qu'il a prise à l'égard de son grand ancêtre. Si Chateaubriand, dans la préface de son Itinéraire, ou Lamartine, dans l'avertissement de son Voyage en Orient, se sentaient encore obligés de mettre en œuvre une subtile stratégie d'autodénigrement pour mieux légitimer leur propre entreprise ${ }^{2 I}$, il n'en est plus de même pour un Gautier, un Hugo ou un Flaubert. À la manière de Sterne, envers lequel leur dette est grande, ils n'hésitent nullement à prendre par le rire leurs distances à l'égard de la doxa viatique en la tournant à son tour en dérision et, par là, à dévoyer le récit de voyage traditionnel:

Avant de commencer le récit de ma triomphante expédition, je crois devoir déclarer à l'univers qu'il ne trouvera ici ni hautes considérations politiques, ni théories sur les chemins de fer, ni plaintes à propos de contrefaçons, ni tirades dithyrambiques en l'honneur des millions au service de toute entreprise dans cet heureux pays de Belgique, véritable Eldorado industriel; il n'y aura exactement dans ma relation que ce que j'aurai vu avec mes yeux, c'est-à-dire avec mon binocle ou avec ma lorgnette, car je craindrais que mes yeux ne me fissent des mensonges. Je n'emprunterai rien au Guide du voyageur, ni aux livres de géographie ou d'histoire, et ceci est un mérite assez rare pour que l'on m'en sache gré. (Gautier, Un tour en Belgique, p. 6)

Vous qui ne voyagez jamais autrement que par l'esprit, allant de livre en livre, de pensée en pensée, et jamais de pays en pays, vous qui passez tous vos étés à l'ombre des mêmes arbres, et tous vos hivers au coin de la même cheminée, vous voulez dès que je quitte Paris, que je vous dise, moi vagabond, à vous solitaire, tout ce que j'ai fait et tout ce que j'ai vu. Soit. J'obéis. Ce que j'ai fait depuis

20. Voir Le Huenen, p. 52 sq., et Montalbetti, p. II sq. Voir aussi Wetzel.

2I. Voir l'analyse qu'en propose J.-Cl. Berchet (p. IO-II). 
avant-hier I8 juillet? Cent cinquante-cinq lieues en trente-six heures. Ce que j'ai vu? J'ai vu Étampes, Orléans, Blois, Tours, Poitiers, et Angoulême. En voulezvous davantage? Vous faut-il des descriptions? Voulez-vous savoir ce que c'est que ces villes, sous quels aspects elles me sont apparues, quel butin d'histoire, d'art et de poésie j'y ai recueilli, chemin faisant, tout ce que j'ai vu enfin? Soit. J'obéis encore. Étampes, c'est une grosse tour entrevue à droite dans le crépuscule au-dessus des toits d'une longue rue [...]. Orléans, c'est une chandelle sur une table ronde dans une salle basse où une fille pâle vous sert un bouillon maigre. Blois, c'est un pont à gauche avec un obélisque pompadour. Le voyageur soupçonne qu'il peut y avoir des maisons à droite, peut-être une ville. [...] Voilà ce que c'est que la France quand on la voit en malle-poste. Que sera-ce lorsqu'on la verra en chemin de fer? (Hugo, Voyages, p. 75I) 22

Le récit de voyage apportait au lecteur la connaissance du monde; avec le romantisme, il lui montre qu'il n'a rien d'autre à connaître qu'une subjectivité, ses aventures, ses impressions, sa quête de soi ou de l'écriture ${ }^{23}$. À ce titre, la recherche permanente du comique fonctionne comme la revendication esthétique si souvent mise en avant par les écrivains voyageurs de la première moitié du XIX ${ }^{\text {e }}$ siècle ${ }^{24}$ : en subvertissant un genre austère, qui les tenait jusqu'alors à distance, ils marquent qu'ils en sont les nouveaux maîtres, à leur manière. Car, à leurs yeux aussi, "voyager doit être un travail sérieux ${ }^{25}$ »... le lecteur dût-il en rire!

\section{BIBLIOGRAPHIE PRIMAIRE}

Nous signalons, entre parenthèses, et quand il y a lieu, la date de première publication de l'ouvrage en volume.

Chateaubriand F.-R. de, Itinéraire de Paris à Jérusalem [I8II] dans Euvres romanesques et voyages, t. 2, M. Regard éd., Paris, Gallimard (Bibliothèque de la Pléiade), I969. Custine A. de, L'Espagne sous Ferdinand VII [I838], J.-F. Tarn éd., Paris, Bourin, I99I. Dumas A., Impressions de voyage en Suisse [1832], Paris, Maspéro, 1982.

Flaubert G. et Du Camp M., Par les champs et par les grèves (posth. : I885 pour les chap. rédigés par Flaubert; 1973 pour ceux rédigés par Du Camp), A. J. Tooke éd., Genève, Droz (Textes littéraires français), 1987

Flaubert G., Correspondance, t. I, J. Bruneau éd., Paris, Gallimard (Bibliothèque de la Pléiade), I973.

22. On pourrait également citer l' "Exposition» des Impressions de voyage en Suisse de Dumas (t. I, p. I-4) ou le début de Par les champs (p. 8I-86), entre autres.

23. On peut voir en effet dans mainte page parodique, pasticheuse ou simplement humoristique de Par les champs, le banc d'essai de Flaubert romancier (voir Debray-Genette, p. 240, 245, 252 sq.)

24. Voir Berchet, p. I2-I3.

25. Lettre de Flaubert à Alfred Le Poittevin (I ${ }^{\text {er }}$ mai I845), Correspondance, p. 226. 
Gautier Th., Un tour en Belgique et Voyage hors barrières [1845] dans Caprices et zigzags, Paris, Charpentier, I884.

Hugo V., Voyages, Cl. Gély éd., dans Euvres complètes, Paris, Laffont (Bouquins), 1987. Léry J. de, Histoire d'un voyage fait en la terre du Brésil (I557), F. Lestringant éd., Presses du Languedoc / Max Chaleil, I992.

Mérimée P., Notes d'un voyage en Corse [1840], P.-M. Auzas éd., Paris, Biro, 1989.

Nerval G. de, Voyage en Orient [185I], dans Euvres, t. 2, H. Lemaître éd., Paris, Garnier, 1958.

Sand G., Lettres d'un voyageur [I837] et Un hiver à Majorque [1842] dans Euvres autobiographiques, t. 2, G. Lubin éd., Paris, Gallimard (Bibliothèque de la Pléiade), I97I.

Stendhal, Mémoires d'un touriste [1838] dans Voyages en France, V. Del Litto éd., Paris, Gallimard (Bibliothèque de la Pléiade), 1992.

Volney C.-F. de, Voyage en Égypte et en Syrie [I787], J. Gaulmier éd., Paris / La Haye, Mouton, 1959 .

\section{BIBLIOGRAPHIE SECONDAIRE}

Berchet J.-Cl., "La préface des récits de voyage au XIX ${ }^{\mathrm{e}}$ siècle», Écrire le voyage, G. Tverdota éd., Paris, Presses de la Sorbonne nouvelle, I994, p. 3-I5.

Bideaux M., "Le voyage littéraire: genèse d'un genre», Littérales, $\mathrm{n}^{\circ} 7$ - «Les modèles du récit de voyage», M.-Chr. Gomez-Géraud éd., Nanterre, université Paris Io, I990, p. I79-I92.

Chupeau J., "Les récits de voyage aux lisières du roman ", Revue d'histoire littéraire de la France, ${ }^{\circ} 77$, 1977, p. 536-553.

Debray-Genette R., "Voyage et description: Par les champs et par les grèves", Métamorphoses du récit, Paris, Seuil, 1988, p. 237-259.

Duchet M., Anthropologie et Histoire au siècle des lumières, Paris, Maspero, I97I.

Guentner W. "Flaubert satiriste dans Par les champs et par les grèves", Voyager en France au temps du romantisme, A. Guyot et Ch. Massol éd., Grenoble, ELLUG, 2003, p. 289-308.

Le Huenen R., «Le récit de voyage, l'entrée en littérature », Études littéraires, n XX-I, Québec, université Laval, 1987, p. 45-6I.

Montalbetti Chr., Le Voyage, le monde et la bibliothèque, Paris, Presses universitaire de France, 1997.

Racault J.-M., "Pour une poétique du prosaïque: le genre du "voyage amusant” ou "voyage littéraire" aux XVII et XVIII siècles", Poésie et voyage. De l'énoncé viatique à l'énoncé poétique, S. Linon-Chipon, V. Magri-Mourgues et S. Moussa éd., La Napoule, La Mancha, 2002, p. 6I-8I.

Sangsue D., "Le récit de voyage humoristique (XVII ${ }^{\mathrm{e}}$-XIX ${ }^{\mathrm{e}}$ siècles) », Revue d'histoire littéraire de la France, n ${ }^{\circ}$ IOI-4, 200I, p. II39-II62.

Wetzel A., "Décrire l'Espagne: référent et réalité dans le récit de voyage littéraire», Standford French Review, n ${ }^{\circ}$ II, 1987, p. 359-373. 